\title{
Contraversive Neglect? A Modulation of Visuospatial Neglect in Association with
} Contraversive Pushing

Nathalie Vaes, $\mathrm{MSc}^{1,2}$, Christophe Lafosse, $\mathrm{PhD}^{2,3}$, Dimitri Hemelsoet, $\mathrm{MD}^{4}$, Els Van Tichelt, $\mathrm{MD}^{5}$, Kristine Oostra, $\mathrm{MD}^{6}$, Guy Vingerhoets, $\mathrm{PhD}^{1}$

${ }^{1}$ Department of Experimental Psychology, Ghent University, Ghent, Belgium

${ }^{2}$ Scientific Unit Rehabilitation Hospital RevArte, Antwerp, Belgium

${ }^{3}$ KU Leuven Department of Psychology, University of Leuven, Leuven, Belgium

${ }^{4}$ Department of Neurology, Ghent University Hospital, Ghent, Belgium

${ }^{5}$ Department of Neurorehabilitation, Rehabilitation Hospital RevArte, Antwerp, Belgium

${ }^{6}$ Department of Physical \& Rehabilitation Medicine, Ghent University Hospital, Ghent, Belgium

Corresponding author:

Nathalie Vaes

Department of Experimental Psychology, Henri Dunantlaan 2, B-9000 Ghent, Belgium

Rehabilitation Hospital RevArte, Drie Eikenstraat 659, B-2650 Edegem, Belgium

Correspondence: nathalie.vaes@ugent.be, nathalie.vaes@ revarte.be 


\begin{abstract}
Objective: Contraversive pushing (CP) is a neurologic disorder characterized by a lateral postural imbalance. Pusher patients actively push towards their contralesional side due to a misperception of the body's orientation in relation to gravity. Although not every patient with CP suffers from spatial neglect $(\mathrm{SN})$, both phenomena are highly correlated in right-hemispheric patients. The present study investigates whether peripersonal visuospatial functioning differs in neglect patients with versus without $\mathrm{CP}\left(\mathrm{NP}^{+}\right.$versus $\mathrm{NP}^{-}$patients). Method: Eighteen right-hemispheric stroke patients with SN were included, of which 17 in a double-blind case-control study and one single case with posterior pushing to supplement the discourse. A computer-based visuospatial navigation task, in which lateralized deviation can freely emerge, was used to quantify visuospatial behavior. In addition, visuospatial orienting was monitored using line bisection. Results: Significant intergroup differences were found. The $\mathrm{NP}^{+}$patients demonstrated a smaller ipsilesional navigational deviation and more cross-over (contralesional instead of ipsilesional deviation) in long line bisection. As such, they demonstrated a contraversive (contralesionally directed) shift in comparison with the $\mathrm{NP}^{-}$patients. Conclusions: These findings highlight the similarity between two systems of space representation. They are consistent with a coherence between the neural processing system which mainly provides for postural control, and the one responsible for non-predominantly postural, visuospatial behavior.
\end{abstract}

Keywords

Visuospatial neglect, contraversive pushing, spatial shift, cross-over, posterior pusher syndrome 


\section{Introduction and Rationale}

Patients with CP demonstrate a 'pusher syndrome', encompassing a contralesionally tilted posture with severe imbalance, an active pushing away from the ipsilesional side with the nonparetic limbs, and resistance to external attempts to correct their posture (Davies, 1985). We refer to Karnath (2007) for a recapitulatory review of this less known phenomenon. CP mainly is reported after stroke, but other cerebral etiologies are possible too (Santos-Pontelli et al., 2004). The critical neural substrates reported so far, are the posterolateral thalamus (Karnath, Ferber, \& Dichgans, 2000a; Karnath, Johannsen, Broetz, \& Kuker, 2005), the insula and postcentral gyrus (Johannsen, Broetz, Naegele, \& Karnath, 2006) (lesion sites) and the inferior frontal gyrus, middle temporal gyrus, precentral gyrus and inferior parietal lobule (Ticini, Klose, Nagele, \& Karnath, 2009) (structurally intact but malperfused). Cases with CP are also described after cerebellar and anterior cerebral artery infarctions, though (Karnath, Suchan, \& Johannsen, 2008; Paci \& Nannetti, 2005). Most authors observed that CP occurs more frequently after right than after left hemisphere lesions (Davies, 2000; Karnath et al., 2000a; Lafosse et al., 2005). In righthemispheric patients, CP often is allied with SN (Bateman \& Riddoch, 1996; Davies, 2000; Lafosse et al., 2005; Saj, Honoré, Coello, \& Rousseaux, 2005). According to Karnath (1994) and hypothesized by Ventre, Flandrin, and Jeannerod (1984), the central transformation of sensory input coordinates to a body centered reference frame is disturbed in SN patients. This induces a horizontal deviation of the spatial reference frame, with a corresponding ipsilesional displacement of the subjective body orientation in the axial plane. In line with this, SN patients' body axis is tilted towards the ipsilesional side, in contrast to the contralesionally tilted body axis in CP patients. Counterintuitively however, Karnath, Ferber, and Dichgans (2000b) found that CP patients' subjective postural vertical was not displaced contralesionally, but distinctly tilted by $18^{\circ}$ towards the ipsilesional side. Other findings exist as well, see Pérennou et al. (2008) concerning a contralesionally perceived vertical in CP. It is suggested that the pusher syndrome stems from a severe misperception of body orientation in relation to gravity, in the coronal plane. Apparently as a pathological compensation mechanism for this misperception, CP patients push their body contraversively (towards the contralesional side) (Karnath, 2007; Karnath et al., $2000 \mathrm{~b}$ ), transferring their center of mass to the contralesional side. On the contrary, the body axis and center of mass of SN patients, reside ipsilesionally (Lafosse, Kerckhofs, Troch, Santens, \& 
Vandenbussche, 2004). In a way of extending this theoretical framework, pushing posteriorly cooccurs in some patients with CP. This peculiar behavior has already been identified early (Davies, 1985). The term "posterior pusher syndrome" was only recently proposed though, denoting a disorder of body orientation in the sagittal plane, characterized by a posterior tilt, pushing the trunk backwards, backward falling and active resistance to corrective attempts to pull the body forward (Cardoen \& Santens, 2010). Based on two case studies, these authors suggest that posterior pushing (PP) occurs on the occasion of progressive, aspecific encephalopathy. However, a casus with PP after a right-hemispheric stroke has been described as well (Mikolajewska, 2012). Interestingly, Santos-Pontelli, Pontes-Neto, and Leite (2011) question whether the PP syndrome is a newly reported neurological entity, or a severe postural reaction to the geriatric 'psychomotor disadaptation syndrome', which is characterized by postural impairments including retropulsion, backward disequilibrium, axial and limb rigidity (Pfitzenmeyer, Mourey, Tavernier, \& Camus, 1999). Backward disequilibrium is associated with a posteriorly positioned center of mass (Mourey, Manckoundia, Martin-Arveux, Tavernier-Vidal, \& Pfitzenmeyer, 2004). Furthermore, a posterior trunk orientation might also be related to a shift in the perception of verticality in the sagittal plane, observed by Utz et al. (2011) in many SN patients.

Given the disturbance in the sensory based central neurologic generation of the bodycentered reference frame in SN, Karnath, Christ, and Hartje (1993) manipulated the proprioceptive input (the head-on-trunk signal) in right-hemispheric SN patients. They observed that $\mathrm{SN}$ decreases by turning the trunk $15^{\circ}$ to the left (real lengthening of the posterior neck muscles) or by vibrating the left posterior neck muscles (apparent lengthening). These results were hypothetically interpreted in line with a contralesional shift in two components necessary for visuomotor coordination and space exploration, namely the subjective midplane localization and the egocentric coordinate system. The trunk midline constitutes the physical anchor for the generation of the egocentric reference frame, allowing the determination of body position with respect to visual space. Hence the spatial orientation of the trunk seems to be determinant for neglecting the contralesional part of space, by dividing space perception into an egocentric "left" and "right" sector (Karnath, Schenkel, \& Fischer, 1991). Intrigued by these studies and the distinct postural characteristics of patients with SN on the one hand and CP on the other, the question sets in about the possibility of a correspondingly distinct pattern of visuospatial 
functioning in these two patient groups. In 2008-2009 we conducted an exploratory investigation (unpublished academic thesis), that nourished the implementation of the current case-control study. It was suggestive of a cross-over phenomenon at long line bisection, in $\mathrm{NP}^{+}$patients. Furthermore, evidence of Honoré, Saj, Bernati, and Rousseaux (2009) thoroughly encouraged more solid research in this respect. Regarding the subjective straight ahead, these authors found a significant contralesional shift in $\mathrm{NP}^{+}$patients, as opposed to the ipsilesional shift in $\mathrm{NP}^{-}$patients. They concluded that the pusher syndrome reverses the ipsilesional orienting bias in SN. To be able to monitor whether right-hemispheric patients' visuospatial orienting and behavior manifest itself differently in $\mathrm{NP}^{+}$versus $\mathrm{NP}^{-}$, we conceived a digital task which allows for quasi unrestricted lateral visuomotor deviation (within the limits of the task surface). This means that patients' responses were not directed towards or triggered by certain stimuli at the left, central or right part, but free to move in a navigation task with complete uniform stimuli across its surface (Vaes et al., 2015). In addition, we wanted to inspect by means of this study, whether cross-over in long line bisection actually is a phenomenon characterizing $\mathrm{NP}^{+}$as opposed to $\mathrm{NP}^{-}$. This should be expected based on the research of Honoré et al. (2009, cf. supra) and Richard and colleagues (Richard, Honoré, Bernati, \& Rousseaux, 2004), the latter of which evinced a significant correlation between long line bisection error and subjective straight ahead position in right-hemispheric SN patients. Indeed, combining both lines of evidence, a reversed long line bisection error -contraversive cross-over- should be observed in association with a reversed subjective straight ahead position in right-hemispheric $\mathrm{NP}^{+}$patients. In place here, is a short demarcation of the cross-over phenomenon in long line bisection hypothesized here, from the one in short line bisection. SN patients can demonstrate cross-over in lines of 2 to $2.5 \mathrm{~cm}$ (Halligan \& Marshall, 1988). Several reasons are advanced for this phenomenon in short lines, such as representational overextension (Ishiai et al., 2004), confabulation released by desinhibition (Chatterjee, 1995; Monaghan \& Shillcock, 1998) and hemianopia (Doricchi et al., 2005). Interesting discussions on hemianopic contralesional line bisection error in longer lines, however, can be found in Kerkhoff and Schenk (2011) and Kuhn et al. (2012a, 2012b). Concerning long line bisection, a spatial performance difference is documented, with less ipsilesional deviation in right, compared to left positioned lines (Heilman \& Valenstein, 1979; Nichelli, Rinaldi, \& Cubelli, 1989). This performance difference fits well with the theory of egocentric space 
representation anchored to the trunk midline, leading to $\mathrm{SN}$ in case of a disturbance in this representational system (Bisiach, Capitani, \& Porta, 1985; Karnath et al., 1991).

\section{Methods}

\section{Research Design}

A multicenter double-blind case-control study was conducted to assess visuospatial functioning in right-hemispheric SN patients with versus without $\mathrm{CP}$. Whenever a patient qualified for inclusion, the Scale for Contraversive Pushing ${ }^{1}$ (SCP, Karnath, Brotz, \& Gotz, 2001; Karnath et al., 2000b) intended for that patient, was given to the collaborating physiotherapist and the visuospatial measurements were administered by the test leader. At the time of testing, patient nor test leader knew whether the patient would be in the $\mathrm{NP}^{+}$or $\mathrm{NP}^{-}$group, and the physiotherapist did not know the patient's visuospatial performance. Patients were only assigned to the $\mathrm{NP}^{+}$group in case of a clinically experienced pusher syndrome by the treating stroke physiotherapist, plus a pusher profile on the SCP, administered by a stroke physiotherapist trained in the use of the SCP. The scale was employed with the detailed instructions published in an update, to enhance its reliability and validity (Karnath \& Brotz, 2007). We adhered to the modified SCP-cutoff criterion of Baccini and coworkers (Baccini, Paci, Nannetti, Biricolti, \& Rinaldi, 2008), being a score $>0$ in each of the three sections of the scale, because of its excellent correspondence with the clinical diagnosis. To avoid confounds due to overlap in features of the $\mathrm{SCP}$, we only included patients in the $\mathrm{NP}^{-}$group if they obtained a zero SCP-score.

The study was approved by the two Committees on Medical Ethics involved, being the ethical committee of the GasthuisZusters Hospitals Antwerp and the leading ethical committee of the University Hospital Ghent.

\footnotetext{
${ }^{1}$ The SCP is based on Davies criteria (Davies, 1985) and assesses the symmetry of the spontaneous posture (related to the contraversive tilt), the extension of the arm or leg to enlarge physical contact with the surface, and the resistance to passive correction of posture, all in the sitting and standing position.
} 


\section{Patients}

For this study, we drew data from the baseline measurements of a recently conducted randomized placebo-controlled trial (results submitted elsewhere). Patients were recruited from the stroke unit (Neurology Department) and the Rehabilitation Center UZ Gent at the Ghent University Hospital, and from Rehabilitation Hospital RevArte (Antwerp). From each center, right-hemispheric stroke patients were considered for trial inclusion after detection of SN signs by the leading neurologist or neurorehabilitation physician, confirmed by a quick screening by means of the Star Cancellation Test (Halligan, Wilson, \& Cockburn, 1990) and a short three-lined bisection task. Upon inclusion, they needed to agree with participation by signing an informed consent. To ascertain a manifest $\mathrm{SN}$ in the patients of the $\mathrm{NP}^{+}$and $\mathrm{NP}^{-}$groups, preventing confounding influences of negligible SN on our depending variables, we opted for secure inclusion criteria based on prior studies: a Center of Cancellation (CoC, Binder, Marshall, Lazar, Benjamin, \& Mohr, 1992) > .081 (Rorden \& Karnath, 2010) on the Bells Test (Gauthier, Dehaut, \& Joanette, 1989), and a mean percentage of deviation of $\geq 14 \%$ (Ferber \& Karnath, 2001) on the Schenkenberg Line Bisection Test (SLBT, Schenkenberg, Bradford, \& Ajax, 1980). Patients were excluded based on the following criteria: declining to participate, significant visual problems, cerebral tumors, traumatic brain injuries, hydrocephalus, comorbid dementia, premorbid mental deterioration, a $>0$ score in only one or two of the three sections of the SCP and not meeting our CoC- or SLBT-criterion. Inferred from previously reported patient numbers in related studies (Honoré et al., 2009; Karnath et al., 2000b; Richard et al., 2004), we deemed a minimum sample size of 15 to 18 included patients to be acceptable, balanced between both groups.

The $\mathrm{NP}^{+}$and $\mathrm{NP}^{-}$groups were comparable in age, sex, handedness, years of education after the age of six, days post-stroke, the presence of hemianopia, and the amount of SN as quantified by the $\mathrm{CoC}$ index at the Bells Test and the mean deviation percentage in the SLBT. Furthermore, their levels of head and gaze deviation, muscle tone at the affected hemi-body, upper extremity impairment and gait independence were similar, suggesting that the severity of their physical post-stroke condition was controlled for too (see the results section 3.1.). 


\section{Descriptive and Outcome Measures}

Next to the conventional demographic characteristics, we assessed a number of descriptives that are informative for the amount of stroke related impairment. They are clarified consecutively. Hemianopia is difficult to objectify in SN (Walker, Findlay, Young, \& Welch, 1991). Besides, the sensitivity of confrontation visual field testing for mild to moderate visual field defects is low. It improves by combining confrontation tests and is rather satisfactory for severe visual defects such as homonymous hemianopia (Kerr, Chew, Eady, Gamble, \& Danesh-Meyer, 2010; Lenworth \& Frank, 1991). Our patients' visual fields were examined by their neurologists and after inclusion by clinical confrontation tests administered by the neuropsychologist, to reduce uncertainty about the presence of hemianopia. The degree of head and gaze deviation was measured by a four level scale ranging from no deviation to a deviation that even cannot be reduced after verbal instruction (Azouvi et al., 2002; Rode, Mauguière, Fischer, \& Boisson, 1995; Rousseaux et al., 2001). For the evaluation of rigidity during passive extension and flexion of the affected fingers, wrist, elbow, knee and ankle, the Ashworth Scale (AS) was used (Ashworth, 1964), a five level scale ranging from no to a severely increased muscle tone. The upper limb motor function was assessed by means of the Utrecht Arm/Hand Test (UAT, Kruitwagen-van Reenen, Post, Mulder-Bouwens, \& Visser-Meily, 2009), an eight level ordinal scale based on the following stages of recovery: a-functional arm, flexion-synergy, first distal selectivity, wrist dorsal flexion, hook grip, cylinder grasp, tweezers grasp and clumsy hand (Brunnstrom, 1966; Twitchell, 1951). To rate the degree of assistance needed while walking, the six ordinal items of the Functional Ambulation Categories (FAC) were employed, ranging from an afunctional gait to independent walking on any surface (Holden, Gill, Magliozzi, Nathan, \& Piehlbaker, 1984).

The outcome measures are twofold. First, we used the navigational terminus and the Center of Navigation (CoN) index of the digital Visuospatial Navigation Test (VNT) of Vaes et al. (2015). Conceived for the use on a wide pen display and existing of symmetrically ordered uniform obstacles (see Figure 1), this test allows for quasi free lateral spatial deviation. The employed pen display has an interactive field of circa 48 to $27 \mathrm{~cm}$ (width to height) and was positioned horizontally in front of the patients. Patients were instructed to start centrally at the bottom of the maze and to find their way (between the obstacles) to the top (the red bar) by the shortest route. The navigational terminus is the end point along the red bar at the top, quantified 
in mm counted from zero, with a maximum value of 233 . With regard to the $\mathrm{X}$-axis of the task, zero is located in the middle. The $\mathrm{CoN}$ index ${ }^{2}$ is the mean percentage of navigational deviation with respect to zero, of all registered pen coordinates at the navigational route, along the $\mathrm{X}$-axis. It is calculated starting from the second white line at the bottom of the maze.

Second, the amount of cross-over in long line bisection was counted, more specifically the number of times that a patient bisected a line left instead of right of its midpoint. The SLBT (Schenkenberg et al., 1980) was used, presented on the same pen display as the one employed for the VNT. Eighteen horizontal lines were taken into account, interlacingly positioned within a left, middle and right test section. Each section contains six lines, always with lengths of 100, 120, 140, 160, 180 and $200 \mathrm{~mm}$. The midpoints of the left and right lines of the same lengths were located at equal distances of the centered line midpoints in the middle section.

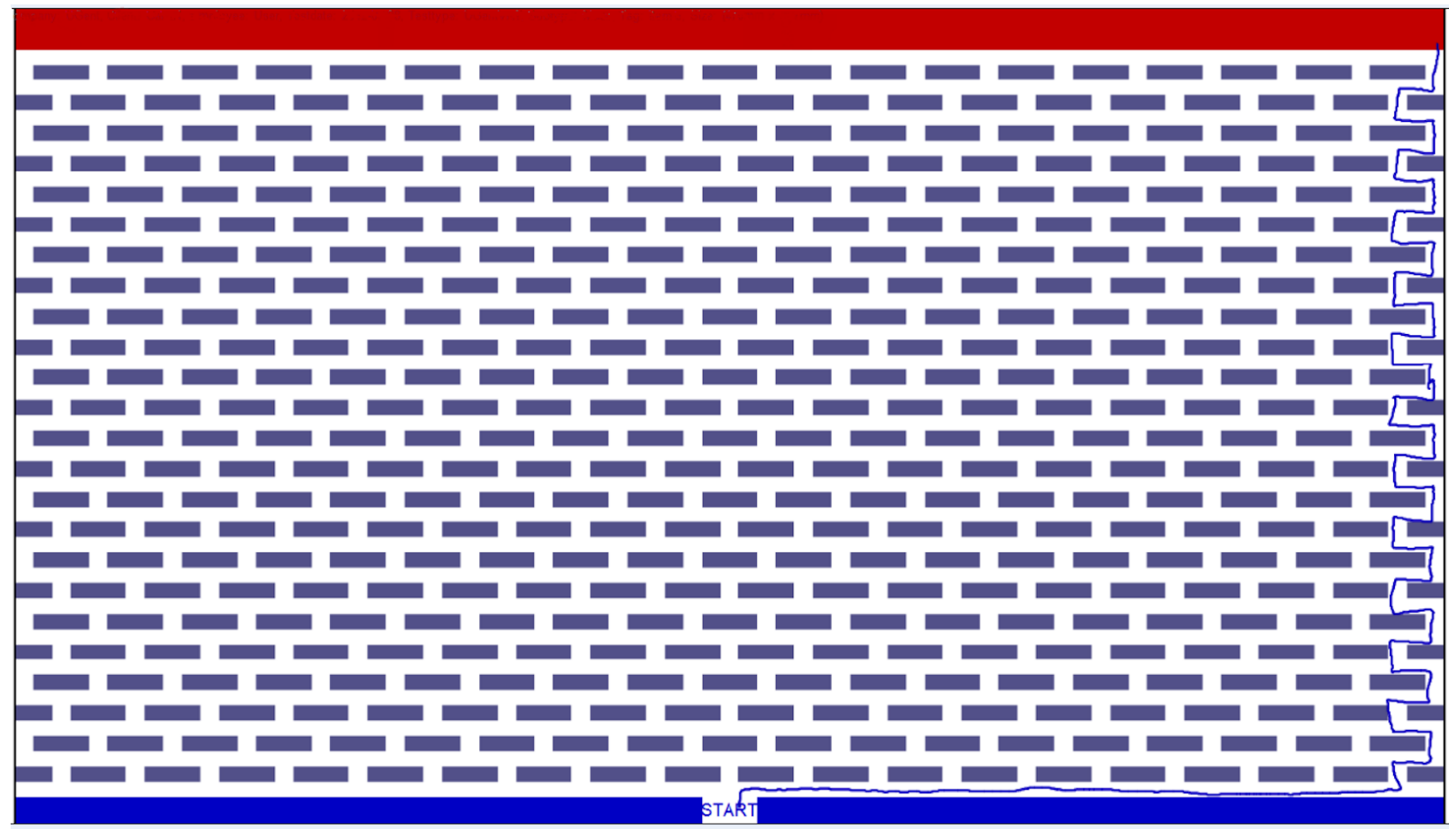

Figure 1. Visuospatial Navigation Test (Vaes et al., 2015), including a typical performance pattern of a clear SN patient, despite the instruction to navigate upwards by the shortest route.

${ }^{2} \operatorname{CoN}=\frac{100}{x_{e}}\left(\frac{1}{n} \sum_{i=1}^{n} x_{i}\right)$, where $x_{i}$ is a pen coordinate at the route, along the $\mathrm{X}$-axis with respect to $0, x_{e}$ the most extreme absolute value a pen coordinate can have along the $\mathrm{X}$-axis, and $n$ the total amount of pen coordinates at the route. 


\section{Research Questions and Statistical Evaluations}

All statistical tests were implemented in IBM SPSS Statistics, version 22. The effects were considered to be significant when the p-values were smaller than .05 . Starting with the quest for equal distributions of the various descriptive characteristics in the $\mathrm{NP}^{+}$and $\mathrm{NP}^{-}$groups, two-sided Kolmogorov-Smirnov Tests were conducted on the multi-valued variables. With regard to the dichotomous variables, two-sided Fisher's Exact Tests were used.

Concerning our first research question, we investigated whether the peripersonal visuospatial behavior of the $\mathrm{NP}^{+}$and $\mathrm{NP}^{-}$groups differed, by analyzing their results of the navigational terminus and the $\mathrm{CoN}$ index from the VNT. Because these variables are bounded and the test results of SN-patients typically are highly skewed, we conducted two-sided MannWhitney $U$ Tests on the data. Complementary to this query, we looked into the visuospatial performance of one patient that rather unexpectedly demonstrated posterior pushing. These data will be described qualitatively instead of analyzed quantitatively, because our current dependent variables do not qualify for measuring changes related to the sagittal plane.

Second, we questioned whether the number of cross-over in long line bisection (SLBT) would be considerably distinct in the $\mathrm{NP}^{+}$and the $\mathrm{NP}^{-}$groups. Additionally, it was inspected whether this would be more pronounced in the left, central or right SLBT section. Because it is not unusual that SN patients skip lines due to general or spatially graded attentional deficits, we needed to take the number of bisected lines into account. For this reason and given that the absence or occurrence of cross-over is a binary event, we modeled the observations with a generalized linear model with a binomial distribution and the logit as link function. To test hypotheses about the model, two-sided Wald Chi-Square Tests were used.

\section{Results}

\section{Patient Flow and Descriptive Characteristics}

After applying the exclusion criteria and after some patient losses due to a sudden discharge or medical deterioration, the case-control study could be implemented in 17 patients. It is 
supplemented with a qualitative single case study of a peculiar casus with PP. Apparently, this patient met the pushing criteria, but they were directed posteriorly instead of contralaterally. We decided not to exclude this case totally, but to record the nature of its navigational visuospatial functioning. The patient flow and counts are represented in Figure 2, as encouraged by the 'Strengthening the Reporting of Observational Studies in Epidemiology' (STROBE) Statement (von Elm et al., 2008). The lesions of the $\mathrm{NP}^{+}$and $\mathrm{NP}^{-}$patients, as inferred from $\mathrm{CT}$ or MRI scans, are middle cerebral artery (MCA) infarctions ( $\mathrm{n}=5$ in $\mathrm{NP}^{+}$and $\mathrm{n}=6$ in $\mathrm{NP}^{-}$, including two in each group with hemorrhagic transformation) or MCA hemorrhages $\left(\mathrm{n}=2\right.$ in $\left.\mathrm{NP}^{+}\right)$, posterior cerebral artery infarction ( $n=1$ in each group) and thalamic hemorrhages ( $n=1$ in each group).

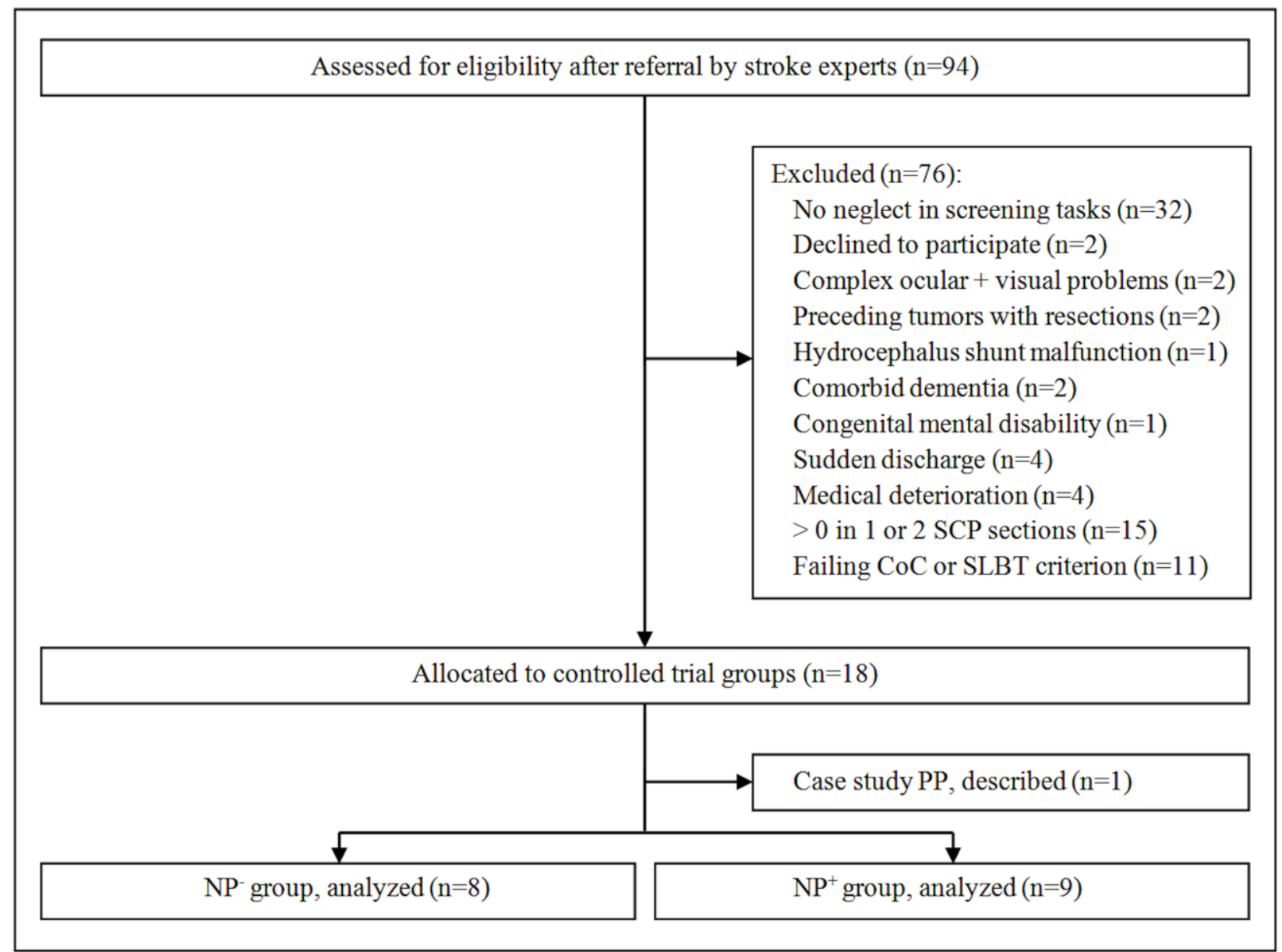

Figure 2. Diagram of the patient flow in the case-control study, as suggested by STROBE. The consecutive phases are the enrollment, group allocation and analyses. Information is provided on the counts and excluded patients. 
Table 1 displays the results of the comparative intergroup analyses regarding the demographic and stroke related descriptives. The $\mathrm{NP}^{+}$and $\mathrm{NP}^{-}$groups did not differ significantly regarding their age, amount of education, sex and handedness. Likewise, the days post-stroke, the presence of hemianopia, the degree of head and gaze deviation, the AS-, UAT- and FAC-scores were not significantly distinct in both groups. The amount of SN as measured by the CoC index at the Bells Test and the mean percentage of SLBT deviation, did not differ significantly between the groups either.

Table 1

Descriptive Characteristics

\begin{tabular}{lccc}
\hline Characteristics & $\begin{array}{c}\mathrm{NP}^{+} \text {group }^{\mathrm{a}} \\
\mathrm{n}=9\end{array}$ & $\mathrm{NP}^{-}$group $^{\mathrm{a}}$ & $\mathrm{n}=8$ \\
\hline Male/female & $6 / 3$ & $4 / 4$ & .637 \\
Handedness R/L & $9 / 0$ & $6 / 2$ & .206 \\
Hemianopia +/- & $3 / 6$ & $2 / 6$ & 1.00 \\
Age & 68.00 & 66.50 & .734 \\
Educational years & 12.00 & 11.50 & 1.00 \\
Days post-stroke & 32.00 & 22.50 & .864 \\
Head and gaze deviation & 2.00 & 1.00 & .167 \\
AS fingers & 1.00 & .50 & .993 \\
AS upper limb (wrist + elbow) & 2.00 & 1.00 & .985 \\
AS lower limb (knee + ankle) & 2.00 & .00 & .189 \\
UAT & .00 & 1.00 & .213 \\
FAC & .00 & 1.00 & .591 \\
CoC Bells Test & .87 & .67 & .780 \\
SLBT mean deviation \% & 14.85 & 39.00 & .146
\end{tabular}

$\mathrm{NP}^{+}$: neglect with contraversive pushing; $\mathrm{NP}^{-}$: neglect without contraversive pushing; AS: Ashworth Scale; UAT: Utrecht Arm/Hand Test; FAC: Functional Ambulation Categories; CoC: Center of Cancellation; SLBT: Schenkenberg Line Bisection Test.

${ }^{\mathrm{a}}$ The values of multi-valued variables represent the medians. 
${ }^{b}$ p-values of the dichotomous variables according to two-sided Fischer's Exact Tests, of the other variables according to two-sided Kolmogorov-Smirnov Tests.

\section{Navigational Visuospatial Behavior}

The medians of the navigational terminus and $\mathrm{CoN}$ index are presented in Figure 3, together with their lower and upper quartiles. The comparative intergroup analyses with respect to these variables revealed significant differences. The p-value concerning the navigational terminus was .011 and the one concerning the $\mathrm{CoN}$ index .027. Figure 4 illuminates this discrepancy in visuospatial behavior between both groups, by depicting their mean navigational routes.
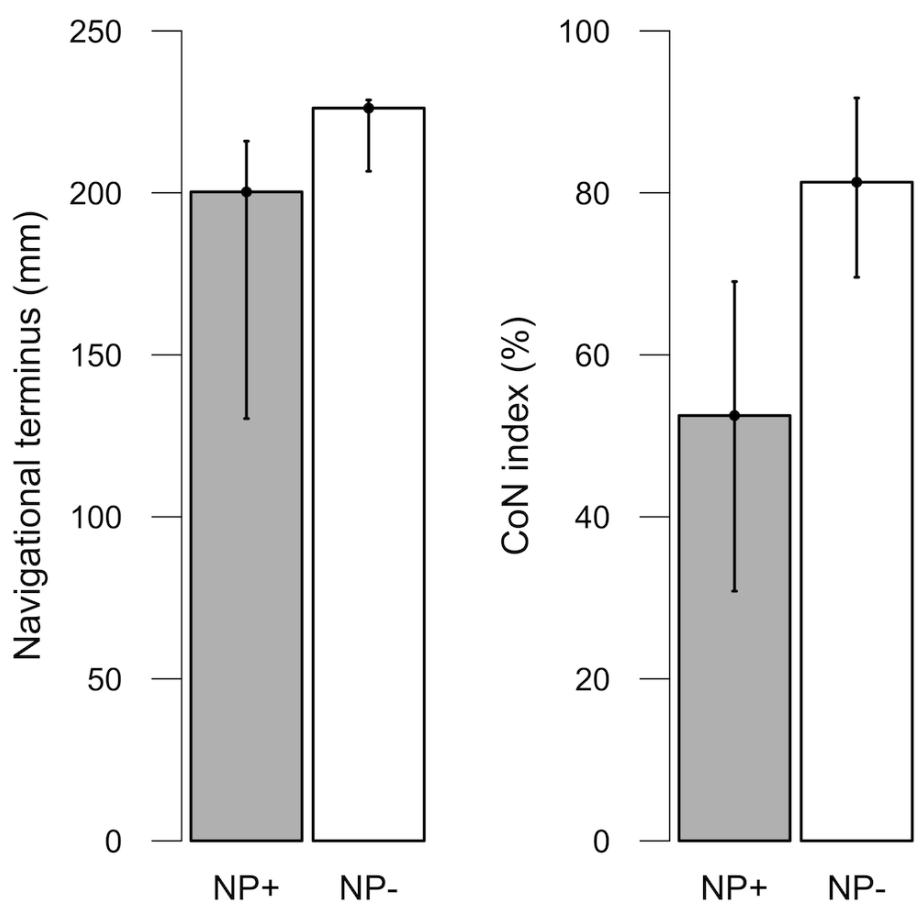

Figure 3. Results of the $\mathrm{NP}^{+}$and $\mathrm{NP}^{-}$groups at both variables of the digital VNT. Navigational terminus $(\mathrm{mm}) \mathrm{NP}^{+}$: median 200.30, lower-upper quartile [130.32-215.95]; Navigational terminus (mm) NP': median 226.15, lower-upper quartile [206.66-228.65]; CoN index (\%) $\mathrm{NP}^{+}$: median 52.50, lower-upper quartile [30.83-69.04]; CoN index (\%) NP': median 81.32 \%, lowerupper quartile [69.58-91.71]. 


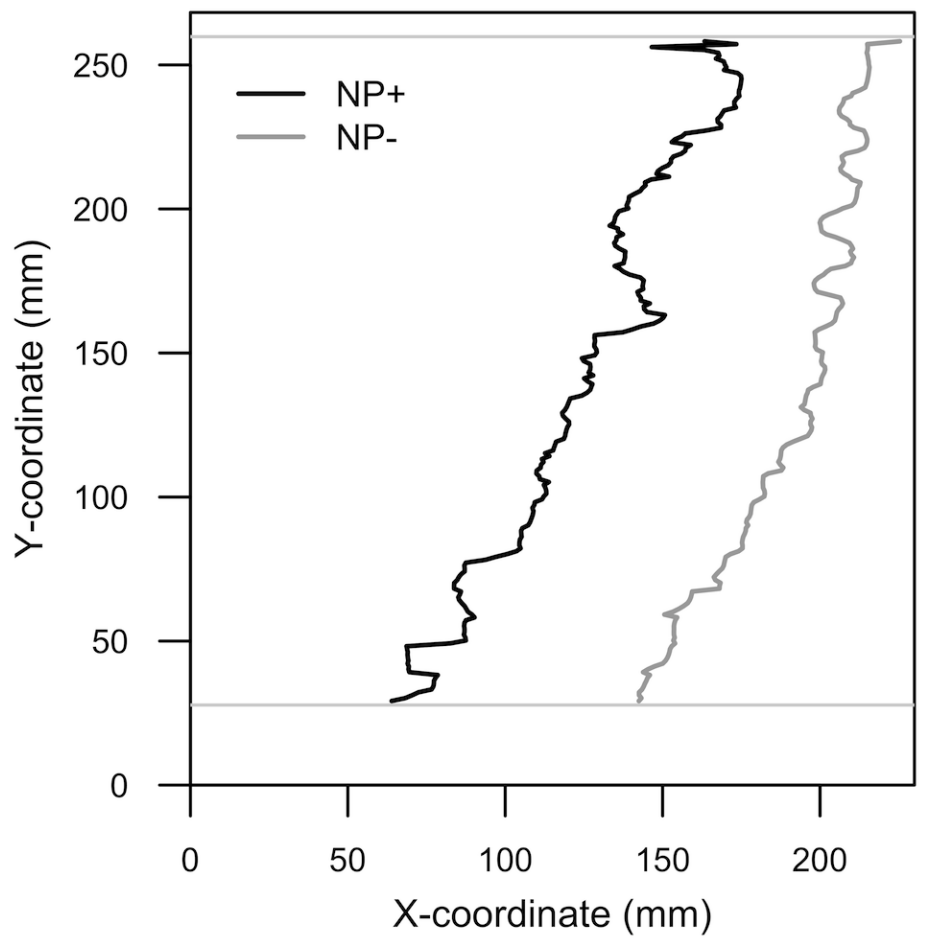

Figure 4. The mean navigational curves of the $\mathrm{NP}^{+}$(left) and $\mathrm{NP}^{-}$(right) groups, visualizing the difference in visuospatial functioning. For representational facilitation, this graph can be superimposed on the right half of the VNT (Vaes et al., 2015). The lower and upper horizontal lines represent the onset (second white line at the bottom of the VNT) and the terminus (the red bar in the VNT) of the measurements. The curves could be reproduced thanks to the storage of the registered pen coordinates in the Metrisquare DiagnoseIS software ${ }^{3}$ running the VNT.

The case demonstrating PP, was a 69-year-old right-handed woman, educationally trained until the age of 14. She suffered from a left hemiplegia and rightward head and gaze deviation after a spontaneous frontoparietal intracerebral hematoma. The post-stroke delay numbered 63 days. Her AS scores were 1 (fingers), 2 (upper limb) and 1 (lower limb). On both the UAT and FAC she scored zero. The CoC of her Bells Test was .91 and the mean SLBT deviation $36.4 \%$.

She could not sit or stand independently, due to a severe posterior tilt and backward pushing with the trunk and legs, especially when the physiotherapist tried to correct her posture forward. Interpreting the SCP posteriorly instead of contraversively, a maximum score was

${ }^{3}$ www.diagnoseis.com, www.metrisquare.com 
reached. At the moment of VNT administration, it first seemed that the patient had forgotten the instruction of navigating upward to the red bar via the shortest route, because she made some backward navigational movements. Therefore, the test leader (for whom the SCP-score was unknown), needed to encourage compliance by repeating the instruction. Then it appeared that moving forward took her some effort, as if the backward direction was a more attractive alternative. Figure 5 demonstrates her task performance.

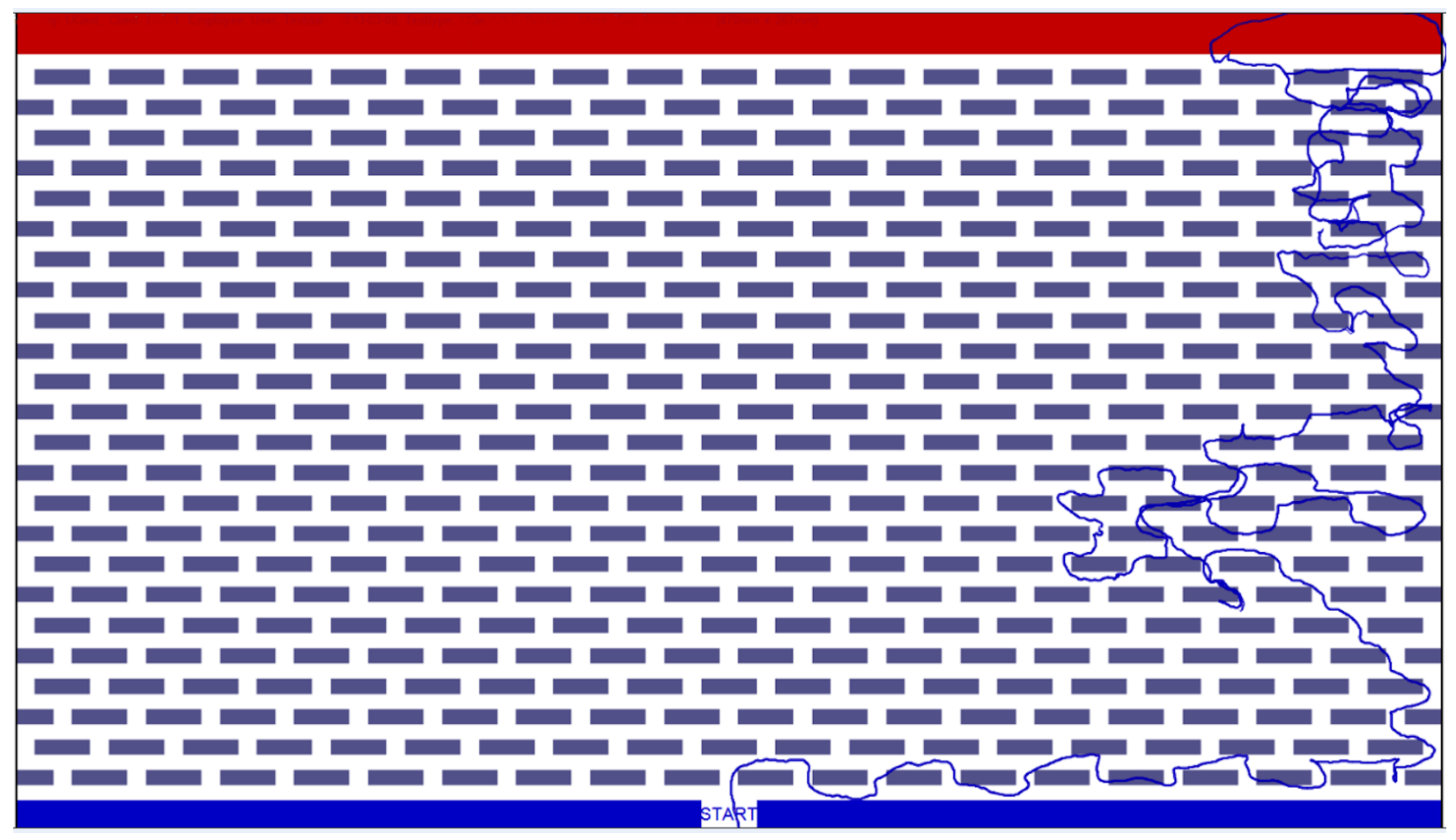

Figure 5. The VNT performed by the casus with posterior pushing.

\section{Cross-over in Long Line Bisection}

The output of the comparative intergroup analyses concerning the number of cross-over in long line bisection is displayed in Table 2. The differences between the $\mathrm{NP}^{+}$and $\mathrm{NP}^{-}$groups are significant concerning the total, the middle and right SLBT section. The patient counts are also displayed, because of some excluded cases in the left and middle subsection, due to absences of trials when all lines of a subsection were neglected. The Wald Chi-Square statistic could not be computed for the left test section, because in none of both groups there was an event of crossover at the left positioned lines. 
Table 2

Comparisons of the number of cross-over in long line bisection between $\mathrm{NP}^{+}$and $\mathrm{NP}^{-}$

\begin{tabular}{ccccccc}
\hline \multicolumn{2}{c}{ SLBT section } & EM Mean & \multicolumn{2}{c}{$95 \%$ Wald CI } & Wald $\chi^{2}$ & p-value \\
& & \multicolumn{7}{c}{ Lower } & Upper & & \\
\hline Total & $\mathrm{NP}^{+}(\mathrm{n}=9)$ & .25 & .18 & .34 & 17.128 & $<.001$ \\
& $\mathrm{NP}^{-}(\mathrm{n}=8)$ & .05 & .02 & .12 & & \\
Left & $\mathrm{NP}^{+}(\mathrm{n}=7)$ & .00 & .00 & .00 & $\mathrm{NA}$ & $\mathrm{NA}$ \\
& $\mathrm{NP}^{-}(\mathrm{n}=6)$ & .00 & .00 & .00 & & \\
Mid & $\mathrm{NP}^{+}(\mathrm{n}=7)$ & .11 & .04 & .26 & 4.500 & .034 \\
& $\mathrm{NP}^{-}(\mathrm{n}=7)$ & .00 & .00 & .00 & & \\
Right & $\mathrm{NP}^{+}(\mathrm{n}=9)$ & .43 & .31 & .57 & 13.808 & $<.001$ \\
& $\mathrm{NP}^{-}(\mathrm{n}=8)$ & .13 & .06 & .25 & &
\end{tabular}

$\mathrm{NP}^{+}$: neglect with contraversive pushing; $\mathrm{NP}^{-}$: neglect without contraversive pushing; SLBT: Schenkenberg Line Bisection Test; EM Mean: estimated marginal mean according to the fitted generalized linear model; CI: confidence interval; NA: non-available (no cross-over in both groups).

\section{Discussion}

The present case-control study reveals a clear difference in peripersonal visuospatial functioning between $\mathrm{NP}^{+}$and $\mathrm{NP}^{-}$patients. In $\mathrm{SN}$ patients with $\mathrm{CP}$, the navigational terminus and Centre of Navigation of the computerized VNT, were significantly shifted towards the contralesional side, compared to the more ipsilesional localization of both variables in SN patients without CP. Additionally, the $\mathrm{NP}^{+}$demonstrated distinctly more cross-over in long line bisection than the $\mathrm{NP}^{-}$ group. Apparently, CP is associated with a contralesionally directed shift in SN behavior, in other words, with 'contraversive neglect'. The contraversive similarity at the postural and visuospatial level hints at a coherence or interaction between the neural processing system for postural control, and the one for non-predominantly postural spatial behavior. Interestingly, this similarity seems not only to be oriented sideward, but also backward. Although this finding is preliminary because it is based on a single case description, our casus with PP also showed signs of a more 
posteriorly directed visuospatial performance at the VNT. The systems for space related body orientation adjustments in the coronal $(\mathrm{CP})$, axial $(\mathrm{SN})$ and sagittal $(\mathrm{PP})$ plane, thus seem to analogously regulate trunk posture and peripersonal visuospatial behavior. It is proposed that human posture in these three planes is controlled by distinct neural networks (Karnath, 2007). The described analogue regulations may suggest that these networks are interconnected through a common neural interface. Furthermore, it is intriguing to draw the following parallel. The decrease in contralesional SN after displacing the trunk to the left by Karnath et al. (1993), could be considered as an experimentally manipulated "compensation" for SN. In parallel, CP can be viewed as a spontaneous (pathological) compensation mechanism attenuating manifest SN, effectuated by the nervous system to accommodate for the dysregulated central transformation of sensory coordinates.

Monaghan and Shillcock (1998) noticed that right displacements in long line bisection turn into left displacements for short lines at the group level, but that there is substantial variability at the individual level. They cite studies were some SN subjects demonstrate cross-over in lines as long as $10 \mathrm{~cm}$, and $20 \mathrm{~cm}$ in one subject. Possibly, characteristics of $\mathrm{CP}$ were present in these patients. It is meaningful for subsequent investigations focusing on $\mathrm{SN}$, to take postural variables into account, because their coherence with other spatial functions can distort or mitigate the nature of SN at the group level. Also in mathematical modeling of spatial neglect, posture should be included. Our SLBT results demonstrated a spatial gradient related to the quantity of crossover in $\mathrm{NP}^{+}$, with a maximum at the right side, less in the middle and no cross-over at the left side. McIntosh, Schindler, Birchall, and Milner (2005) presented a mathematical approach based on the weightings of line endpoints in determining the bisection response. Their endpoint weighting analysis can account for the effects of line length and spatial position on bisection error, including cross-over. The asymmetry in the endpoint weightings in patients with SN, called the 'endpoint weighting bias' (EWB), is measured by the difference between the right and left endpoint weightings. They hypothesized that the EWB could be considered as a measure of lateral attentional bias, in which the right endpoint outcompetes the left one for limited attentional resources. Following their quantitative model, the bisection error can be predicted by subtracting the peripersonal location of the line midpoint, from the sum of the weighted left and right endpoints plus a regression constant $k$. The authors acknowledge that a theoretical interpretation of $k$ still needs to be proposed. We suggest that the constant $k$ might be significantly impacted by 
interindividual differences in the orientation of the trunk midline or the egocentric reference frame. It would be an appealing endeavor to inspect whether these variables can predict the response position for a given stimulus to a higher degree. Consequently, in this way the model should also be able to distinguish between $\mathrm{NP}^{+}$and $\mathrm{NP}^{-}$patients.

The observations in $\mathrm{NP}^{+}$patients, of a spatial gradient related to the quantity of cross-over and a contralesionally directed shift in the VNT, puts forward the inquiry about ipsilesional neglect'. It should be investigated whether contraversive neglect emanates from a corrective neural reorganization in the egocentric reference frame, leading to less contralesional neglect thanks to a constructive compensation mechanism. The alternative would be a pathological compensation mechanism for their posturospatial bias, averting patients away from their ipsilesional side. As such, the field of their attentional and motor behavior would be narrowed due to their contralesional, plus a quantum of ipsilesional neglect.

An important issue to deal with in future studies, is the underlying mechanism of the currently reported observations. Central neurologic causal mechanisms that are theorized for CP are a misperception of verticality at the body oriented gravitational (Karnath et al., 2000b), or transmodal level (Pérennou et al., 2008). The central neurologic origin of contraversive neglect in association with $\mathrm{CP}$ has to be ensured. If factors such as ipsilesional hypertonia or rigidity are more pronounced in patients with $\mathrm{CP}$, it is not inconceivable that they experience more movement constraints than patients without CP. Even the more contralesionally tilted trunk orientation could lead to less ipsilesionally elongated arm movements in $\mathrm{NP}^{+}$compared to $\mathrm{NP}^{-}$ patients. However, these potential peripheral motor causes are less likely, because ipsilesionally extended arm movements are surely present in the $\mathrm{NP}^{+}$patients to enlarge their physical support base. Additionally, it will be extremely interesting to unravel with refined paradigms, whether contraversive neglect is primarily encountered at the motor or at the perceptual level, or at the attentional and representational level as well. An excellent example would be to investigate whether similar performance differences between $\mathrm{NP}^{+}$and $\mathrm{NP}^{-}$emerge in a spatial task at least as sensitive as the VNT, but on the representational level and requiring verbal responses, in analogy with the Piazza Del Duomo experiment (Bisiach \& Luzzatti, 1978) or the verbal Landmark Task (Bisiach, Ricci, Lualdi, \& Colombo, 1998). Similar research questions can be formulated regarding the personal or extrapersonal dimensions (instead of the peripersonal one as discussed here) and regarding other sensory modalities than the visual one. Finally, robust brain imaging 
studies in $\mathrm{NP}^{+}$and $\mathrm{NP}^{-}$patients will be of great relevance to unravel to quest for the underlying neural mechanism, preferably by voxel-based lesion-symptom mapping (Bates et al., 2003).

Unraveling these issues does not only advance the theoretical understanding of the coherence between different neural processing systems of space representation. It is of practical relevance as well, by complementing neurorehabilitation and refining neuropsychological diagnostics. Specific interventions for CP rehabilitation are proposed already, using visual feedback to correct body orientation (Broetz, Johannsen, \& Karnath, 2004; Broetz \& Karnath, 2005), taking into account the spatial body misperception and fear of falling (Shepherd \& Carr, 2005), learning compensation strategies through vocal and visual feedback (Paci \& Nannetti, 2004) and forced control of upright position in machine-supported gait training (Krewer et al., 2013). Gaining more insight into the contraversive shift in spatial behavior of $\mathrm{NP}^{+}$patients can contribute to targeted interventions, by integrating the findings into a holistic rehabilitation approach.

The present observations of peripersonal visuospatial contraversive neglect in patients with $\mathrm{CP}$, can encourage larger controlled trials to investigate this topic in greater depth. Additionally, they foster nuanced diagnostics of $\mathrm{SN}$ in $\mathrm{CP}$ and advocate targeted posturo- and visuospatial rehabilitation.

\section{Acknowledgements}

We sincerely thank Gudrun Nys, $\mathrm{PhD}$, for the research opportunities. We like to express our gratitude to all patients who contributed to the study. We are also very thankful to the collaborating physiotherapists, for concisely administering the SCP in our participants. This project was supported by Grant G.0906.10N from the Fund for Scientific Research Flanders (FWO-Vlaanderen), Belgium. The authors declare that they have no conflicts of interest. 


\section{References}

Ashworth, B. (1964). Preliminary trial of carisoprodol in multiple sclerosis. Practitioner, 192, 540-542.

Azouvi, P., Samuel, C., Louis-Dreyfus, A., Bernati, T., Bartolomeo, P., Beis, J. M., . . . Rousseaux, M. (2002). Sensitivity of clinical and behavioural tests of spatial neglect after right hemisphere stroke. Journal of Neurology Neurosurgery and Psychiatry, 73(2), 160166. doi: DOI 10.1136/jnnp.73.2.160

Baccini, M., Paci, M., Nannetti, L., Biricolti, C., \& Rinaldi, L. A. (2008). Scale for Contraversive Pushing: Cutoff scores for diagnosing "Pusher Behavior" and construct validity. Physical Therapy, 88(8), 947-955. doi: 10.2522/Ptj.20070179

Bateman, A., \& Riddoch, M. J. (1996). Neuropsychological perspectives on "pusher syndrome". European Journal of Phyical Medicine and Rehabilitation, 6(3), 93-96.

Bates, E., Wilson, S. M., Saygin, A. P., Dick, F., Sereno, M. I., Knight, R. T., \& Dronkers, N. F. (2003). Voxel-based lesion-symptom mapping. Nature Neuroscience, 6, 448-450. doi: $10.1038 / \mathrm{nn} 1050$

Binder, J., Marshall, R., Lazar, R., Benjamin, J., \& Mohr, J. P. (1992). Distinct syndromes of hemineglect. Archives of Neurology, 49(11), 1187-1194.

Bisiach, E., Capitani, E., \& Porta, E. (1985). Two basic properties of space representation in the brain: evidence from unilateral neglect. Journal of Neurology Neurosurgery and Psychiatry, 48(2), 141-144. doi: 10.1136/jnnp.48.2.141

Bisiach, E., \& Luzzatti, C. (1978). Unilateral neglect of representational space. Cortex, 14(1), 129-133. doi: 10.1016/S0010-9452(78)80016-1

Bisiach, E., Ricci, R., Lualdi, M., \& Colombo, M. R. (1998). Perceptual en response bias in unilateral neglect: Two modified versions of the Milner Landmark task. Brain and Cognition, 37(3), 369-386. doi: 10.1006/brcg.1998.1003

Broetz, D., Johannsen, L., \& Karnath, H. O. (2004). Time course of 'pusher syndrome' under visual feedback treatment. Physiotherapy Research International, 9(3), 138-343.

Broetz, D., \& Karnath, H. O. (2005). New aspects for the physiotherapy of pushing behaviour. Neurorehabilitation, 20(2), 133-138. 
Brunnstrom, S. (1966). Motor testing procedures in hemiplegia: based on sequential recovery stages. Physical Therapy, 46(4), 357-375.

Cardoen, S., \& Santens, P. (2010). Posterior pusher syndrome: A report of two cases. Clinical Neurology and Neurosurgery, 112(4), 347-349. doi: 10.1016/j.clineuro.2009.12.007

Chatterjee, A. (1995). Cross-over, Completion and Confabulation in Unilateral Spatial Neglect. Brain, 118, 455-465. doi: 10.1093/brain/118.2.455

Davies, P. M. (1985). Steps to follow. A guide to the treatment of adult hemiplegia. New York: Springer.

Davies, P. M. (2000). Steps to follow. A guide to the treatment of adult hemiplegia. New York: Springer.

Doricchi, F., Guariglia, P., Figliozzi, F., Silvetti, M., Bruno, G., \& Gasparini, M. (2005). Causes of cross-over in unilateral neglect: between-group comparisons, within-patient dissociations and eye movements. Brain, 128, 1386-1406. doi: 10.1093/Brain/Awh461

Ferber, S., \& Karnath, H. O. (2001). How to assess spatial neglect - Line bisection or cancellation tasks? Journal of Clinical and Experimental Neuropsychology, 23(5), 599-607. doi: 10.1076/jcen.23.5.599.1243

Gauthier, L., Dehaut, F., \& Joanette, Y. (1989). The Bells Test: A quantitative and qualitative test for visual neglect. International Journal of Clinical Neuropsychology, 11(2), 49-54.

Halligan, P., Wilson, B., \& Cockburn, J. (1990). A short screening test for visual neglect in stroke patients. International Disability Studies, 12(3), 95-99.

Halligan, P. W., \& Marshall, J. C. (1988). How long is a piece of string? A study of line bisection in a case of visual neglect. Cortex, 24(2), 321-328.

Heilman, K. M., \& Valenstein, E. (1979). Mechanisms underlying hemispatial neglect. Annals of Neurology, 5(2), 166-170. doi: 10.1002/ana.410050210

Holden, M. K., Gill, K. M., Magliozzi, M. R., Nathan, J., \& Piehlbaker, L. (1984). Clinical gait assessment in the neurologically impaired - Reliability and meaningfulness. Physical Therapy, 64(1), 35-40.

Honoré, J., Saj, A., Bernati, T., \& Rousseaux, M. (2009). The pusher syndrome reverses the orienting bias caused by spatial neglect. Neuropsychologia, 47(3), 634-638. doi: 10.1016/j.neuropsychologia.2008.11.008 
Ishiai, S., Koyama, Y., Nakano, N., Seki, K., Nishida, Y., \& Hayashi, K. (2004). Image of a line is not shrunk but neglected - Absence of crossover in unilateral spatial neglect. Neuropsychologia, 42(2), 251-256. doi: 10.1016/S0028-3932(03)00153-2

Johannsen, L., Broetz, D., Naegele, T., \& Karnath, H. O. (2006). "Pusher syndrome" following cortical lesions that spare the thalamus. Journal of Neurology, 253(4), 455-463. doi: $10.1007 / \mathrm{s} 00415-005-0025-7$

Karnath, H. O. (1994). Disturbed coordinate transformation in the neural representation of space as the crucial mechanism leading to neglect. Neuropsychological Rehabilitation, 4(2), 147-150. doi: 10.1080/09602019408402273

Karnath, H. O. (2007). Pusher Syndrome - a frequent but little-known disturbance of body orientation perception. Journal of Neurology, 254(4), 415-424. doi: 10.1007/s00415-0060341-6

Karnath, H. O., \& Brotz, D. (2007). Instructions for the Clinical Scale for Contraversive Pushing (SCP). Neurorehabilitation and Neural Repair, 21(4), 370-371. doi: $10.1177 / 1545968307300702$

Karnath, H. O., Brotz, D., \& Gotz, A. (2001). Clinical symptoms, origin, and therapy of the ,pusher syndrome". Nervenarzt, 72(2), 86-92. doi: 10.1007/s001150050719

Karnath, H. O., Christ, K., \& Hartje, W. (1993). Decrease of contralateral neglect by neck muscle vibration and spatial orientation of trunk midline. Brain, 116, 383-396. doi: 10.1093/brain/116.2.383

Karnath, H. O., Ferber, S., \& Dichgans, J. (2000a). The neural representation of postural control in humans. Proceedings of the National Academy of Sciences of the United States of America, 97(25), 13931-13936. doi: 10.1073/pnas.240279997

Karnath, H. O., Ferber, S., \& Dichgans, J. (2000b). The origin of contraversive pushing: evidence for a second graviceptive system in humans. Neurology, 55(9), 1298-1304.

Karnath, H. O., Johannsen, L., Broetz, D., \& Kuker, W. (2005). Posterior thalamic hemorrhage induces "pusher syndrome". Neurology, 64(6), 1014-1019. doi: 10.1212/01.WNL.0000154527.72841.4A

Karnath, H. O., Schenkel, P., \& Fischer, B. (1991). Trunk orientation as the determining factor of the contralateral deficit in the neglect syndrome and as the physical anchor of the internal 
representation of body orientation in space. Brain, 114, 1997-2014. doi: 10.1093/brain/114.4.1997

Karnath, H. O., Suchan, J., \& Johannsen, L. (2008). Pusher syndrome after ACA territory infarction. European Journal of Neurology, 15(8), e84-85. doi: 10.1111/j.14681331.2008.02187.x

Kerkhoff, G., \& Schenk, T. (2011). Line bisection in homonymous visual field defects - Recent findings and future directions. Cortex, 47(1), 53-58. doi: 10.1016/j.cortex.2010.06.014

Kerr, N. M., Chew, S. S. L., Eady, E. K., Gamble, G. D., \& Danesh-Meyer, H. V. (2010). Diagnostic accuracy of confrontation visual field tests. Neurology, 74(15), 1184-1190. doi: 10.1212/WNL.0b013e3181d90017

Krewer, C., Riess, K., Bergmann, J., Muller, F., Jahn, K., \& Koenig, E. (2013). Immediate effectiveness of single-session therapeutic interventions in pusher behaviour. Gait \& Posture, 37(2), 246-250. doi: 10.1016/j.gaitpost.2012.07.014

Kruitwagen-van Reenen, E. T., Post, M. W. M., Mulder-Bouwens, K., \& Visser-Meily, J. M. A. (2009). A simple bedside test for upper extremity impairment after stroke: Validation of the Utrecht Arm/Hand Test. Disability and Rehabilitation, 31(16), 1338-1343. doi: $10.1080 / 09638280902846855$

Kuhn, C., Bublak, P., Jobst, U., Rosenthal, A., Reinhart, S., \& Kerkhoff, G. (2012a). Contralesional spatial bias in chronic hemianopia: the role of $(\mathrm{ec})$ centric fixation, spatial cueing and visual search. Neuroscience, 210, 118-127. doi: 10.1016/J.Neuroscience.2012.03.020

Kuhn, C., Rosenthal, A., Bublak, P., Grotemeyer, K.H., Reinhart, S., \& Kerkhoff, G. (2012b). Does spatial cueing affect line bisection in chronic hemianopia? Neuropsychologia, 50(7), 1656-1662. doi: 10.1016/J.Neuropsychologia.2012.03.021

Lafosse, C., Kerckhofs, E., Troch, M., Santens, P., \& Vandenbussche, E. (2004). Graviceptive misperception of the postural vertical after right hemisphere damage. Neuroreport, 15(5), 887-891.

Lafosse, C., Kerckhofs, E., Troch, M., Vereeck, L., Van Hoydonck, G., Moeremans, M., . . . Vandenbussche, E. (2005). Contraversive pushing and inattention of the contralesional hemispace. Journal of Clinical and Experimental Neuropsychology, 27(4), 460-484. doi: $10.1080 / 13803390490520463$ 
Lenworth, N. J., \& Frank, G. B. (1991). The accuracy of confrontation visual field test in comparison with automated perimetry. Journal of the National Medical Association, 83(10), 895-898.

McIntosh, R. D., Schindler, I., Birchall, D., \& Milner, A. D. (2005). Weights and measures: A new look at bisection behaviour in neglect. Cognitive Brain Research, 25(3), 833-850. doi: 10.1016/j.cogbrainres.2005.09.008

Mikolajewska, E. (2012). Posterior pusher syndrome - case report. Central European Journal of Medicine, 7(3), 354-357. doi: 10.2478/s11536-011-0145-7

Monaghan, P., \& Shillcock, R. (1998). The cross-over effect in unilateral neglect. Modelling detailed data in the line-bisection task. Brain, 121(5), 907-921.

Mourey, F., Manckoundia, P., Martin-Arveux, I., Tavernier-Vidal, B., \& Pfitzenmeyer, P. (2004). Psychomotor disadaptation syndrome - A new clinical entity in geriatric patients. Geriatrics, 59(5), 20-24.

Nichelli, P., Rinaldi, M., \& Cubelli, R. (1989). Selective spatial attention and length representation in normal subjects and in patients with unilateral spatial neglect. Brain and Cognition, 9(1), 57-70. doi: 10.1016/0278-2626(89)90044-4

Paci, M., \& Nannetti, L. (2004). Physiotherapy for pusher behaviour in a patient with post-stroke hemiplegia. Journal of Rehabilitation Medicine, 36(4), 183-185. doi: $10.1080 / 16501970410029762$

Paci, M., \& Nannetti, L. (2005). The pusher syndrome in a patient with cerebellar infarction. Physiotherapy Research International, 10(3), 176-177.

Pérennou, D. A., Mazibrada, G., Chauvineau, V., Greenwood, R., Rothwell, J., Gresty, M. A., \& Bronstein, A. M. (2008). Lateropulsion, pushing and verticality perception in hemisphere stroke: a causal relationship? Brain, 131(9), 2401-2413. doi: 10.1093/brain/awn170

Pfitzenmeyer, P., Mourey, F., Tavernier, B., \& Camus, A. (1999). Psychomotor desadaptation syndrome. Archives of Gerontology and Geriatrics, 28(3), 217-225. doi: 10.1016/S01674943(99)00005-9

Richard, C., Honoré, J., Bernati, T., \& Rousseaux, M. (2004). Straight-ahead pointing correlates with long-line bisection in neglect patients. Cortex, 40(1), 75-83. doi: 10.1016/S00109452(08)70921-3 
Rode, G., Mauguière, F., Fischer, C., \& Boisson, D. (1995). Lésions hémisphériques droites et négligence unilatérale; La part de la déafférentation. Annales de Réadaptation et de Médecine Physique, 38, 324.

Rorden, C., \& Karnath, H. O. (2010). A simple measure of neglect severity. Neuropsychologia, 48(9), 2758-2763. doi: 10.1016/j.neuropsychologia.2010.04.018

Rousseaux, M., Beis, J. M., Pradat-Diehl, P., Martin, Y., Bartolomeo, P., Bernati, T., . . Azouvi, P. (2001). Présentation d'une batterie de dépistage de la négligence spatiale. Normes et effets de l'âge, du niveau d'éducation, du sexe, de la main et de la latéralité. Revue Neurologique, 157, 1385-1400.

Saj, A., Honoré, J., Coello, Y., \& Rousseaux, M. (2005). The visual vertical in the pusher syndrome - Influence of hemispace and body position. Journal of Neurology, 252(8), 885891. doi: 10.1007/s00415-005-0716-0

Santos-Pontelli, T. E. G., Pontes-Neto, O. M., Colafemina, J. F., de Araujo, D. B., Santos, A., \& Leite, J. P. (2004). Contraversive pushing in non-stroke patients. Journal of Neurology, 251(11), 1324-1328. doi: 10.1007/s00415-004-0532-y

Santos-Pontelli, T. E. G., Pontes-Neto, O. M., \& Leite, J. P. (2011). 'Posterior pusher syndrome' or 'psychomotor disadaptation syndrome'? Clinical Neurology and Neurosurgery, 113(6), 521-521. doi: 10.1016/j.clineuro.2011.01.012

Schenkenberg, T., Bradford, D. C., \& Ajax, E. T. (1980). Line bisection and unilateral visual neglect in patients with neurologic impairment. Neurology, 30(5), 509-517.

Shepherd, R. B., \& Carr, J. (2005). New aspects for the physiotherapy of pushing behaviour, D. Broetz and H.-O. Karnath, Neurorehabilitation 20 (2005),133-138. Neurorehabilitation, 20(4), 343-345.

Ticini, L. F., Klose, U., Nagele, T., \& Karnath, H. O. (2009). Perfusion imaging in Pusher syndrome to investigate the neural substrates involved in controlling upright body position. Plos One, 4(5), e5737. doi: 10.1371/journal.pone.0005737

Twitchell, T. E. (1951). The restoration of motor function following hemiplegia in man. Brain, 74(4), 443-480.

Utz, K. S., Keller, I., Artinger, F., Stumpf, O., Funk, J., \& Kerkhoff, G. (2011). Multimodal and multispatial deficits of verticality perception in hemispatial neglect. Neuroscience, 188, 68-79. doi: 10.1016/J.Neuroscience.2011.04.068 
Vaes, N., Lafosse, C., Nys, G., Schevernels, H., Dereymaeker, L., Oostra, K., Hemelsoet, D., \& Vingerhoets, G. (2015). Capturing peripersonal spatial neglect: An electronic method to quantify visuospatial processes. Behavior Research Methods, 47, 27-44. doi: 10.1037/neu0000205

Ventre, J., Flandrin, J. M., \& Jeannerod, M. (1984). In search for the egocentric reference. A neurophysiological hypothesis. Neuropsychologia, 22(6), 797-806.

von Elm, E., Altman, D. G., Egger, M., Pocock, S. J., Gotzsche, P. C., Vandenbroucke, J. P., \& Initiative, S. (2008). The Strengthening the Reporting of Observational Studies in Epidemiology (STROBE) statement: guidelines for reporting observational studies. Journal of Clinical Epidemiology, 61(4), 344-349. doi: 10.1016/j.jclinepi.2007.11.008

Walker, R., Findlay, J. M., Young, A. W., \& Welch, J. (1991). Disentangling neglect and hemianopia. Neuropsychologia, 29(10), 1019-1027. doi: 10.1016/0028-3932(91)90065-G 